\title{
Dangguijakyak-san ameliorates memory deficits in ovariectomized mice by upregulating hippocampal estrogen synthesis
}

Deok-Sang Hwang ${ }^{1}$, Namkwon Kim², Jin Gyu Choi ${ }^{2}$, Hyo Geun Kim³ ${ }^{3}$, Hocheol Kim ${ }^{4}$ and Myung Sook Oh ${ }^{2,3^{*}}$

\begin{abstract}
Background: Dangguijakyak-san (DJS) is an herbal formulation that has been clinically applicable for treating postmenopausal symptoms and neurological disorders. It is reported that hippocampal estrogen attenuates memory impairment via neuroprotection and synaptogenesis. However, the effect of DJS on hippocampal estrogen synthesis remains unknown. In this study, we explored the effect of DJS and its neuroprotective mechanism against memory impairment in ovariectomized (OVX) mice, with respect to hippocampal estrogen stimulation.

Methods: Cell cultures were prepared from the hippocampi of 18-day-old embryos from timed pregnant Sprague-Dawley rats. The hippocampi were dissected, collected, dissociated, and plated in 60-mm dishes. The cells were treated with DJS for $48 \mathrm{~h}$ and the supernatant was collected to determine estrogen levels. Female ICR mice (8-weeks-old) were housed for 1 week and ovariectomy was performed to remove the influence of ovary-synthesized estrogens. Following a 2-week post-surgical recovery period, the mice were administrated with DJS (50 and $100 \mathrm{mg} / \mathrm{kg} /$ day, p.o.) or $17 \beta$-estradiol (200 $\mu \mathrm{g} / \mathrm{kg} /$ day, i.p.) once daily for 21 days. Hippocampal and serum estrogen levels were determined using enzyme-linked immunosorbent assay kit. Memory behavioral tests, western blot, and immunohistochemical analyses were performed to evaluate the neuroprotective effects of DJS in this model.
\end{abstract}

Results: DJS treatment promoted estrogen synthesis in primary hippocampal cells and the hippocampus of OVX mice, resulting in the amelioration of OVX-induced memory impairment. Hippocampal estrogen stimulated by DJS treatment contributed to the activation of CAMP response element-binding protein and synaptic protein in OVX mice.

Conclusion: DJS may attenuate memory deficits in postmenopausal women via hippocampal estrogen synthesis.

Keywords: Dangguijakyak-san, Hippocampal estrogen, Memory, Ovariectomy

\footnotetext{
* Correspondence: msohok@khu.ac.kr

${ }^{2}$ Department of Life and Nanopharmaceutical Sciences, Graduate School,

Kyung Hee University, 26, Kyungheedae-ro, Dongdaemun-gu, Seoul 02447,

Republic of Korea

${ }^{3}$ Department of Oriental Pharmaceutical Science, College of Pharmacy and

Kyung Hee East-West Pharmaceutical Research Institute, Kyung Hee

University, 26, Kyungheedae-ro, Dongdaemun-gu, Seoul 02447, Republic of

Korea

Full list of author information is available at the end of the article
} 


\section{Background}

It is well known that estrogen plays an indispensable role in cognitive function [1]. Estrogen regulates the dendritic spine density and length in various regions of the brain and enhances long-term potentiation (LTP) at the CA3-CA1 synapse [2-5] via both cell nuclear and membrane receptors [6, 7]. Many studies have reported the molecular mechanisms associated with memoryenhancing effects of estrogen. Estrogen regulates the expression of essential extracellular-signal-regulated kinase (ERK)/cyclic-AMP response element-binding protein (CREB) signaling in long-term hippocampal memory in neurons [8-11] and synaptic protein, including synaptophysin (SYN) and postsynaptic density protein-95 (PSD95) [12-14]. Generally, estrogen is regarded as being synthesized in the ovary and transported to the brain via the blood-brain-barrier [15-17]. Interestingly, it was reported that estrogen is synthesized from cholesterol and androgen precursor $[18,19]$ by neurons and glia in numerous brain regions including the hippocampus, hypothalamus, and cerebral cortex [20-22]. Even though the relative contributions of ovary-synthesized estrogen and brain-synthesized estrogen in memory function have not yet been fully elucidated, it is reported that brainsynthesized estrogen has an important role in memory formation by the preservation of hippocampal synapses [23-25]. Therefore, stimulating estrogen synthesis in the hippocampus will be a new strategy for attenuating memory impairment due to ovary dysfunction status at the postmenopausal stage.

Dangguijakyak-san (DJS), also called Tokishakuyakusan in Japan and Danggui-Shaoyao-san in China, is a traditional and popular herbal formula that consists of six traditional herbs and is widely used in neuro-associated disorders after menopause in women [26]. Many studies have demonstrated that DJS is involved in memory functions: DJS enhances LTP by ERK/CREB/brain-derived neurotrophic factor cascade in the hippocampus [27]. It ameliorates memory impairment and increases neurogenesis through modulating the Akt/glycogen synthase kinase- 3 beta signaling in the bilateral common carotid artery occlusion-induced ischemia model [28]. Administering DJS and its fraction ameliorate cognitive dysfunction caused by amyloid beta in mice via decreasing the contents and deposition of amyloid beta [29]. Additionally, Toriizuka $\mathrm{K}$ et al. reported that DJS improved memory in ovariectomized (OVX) mice via regulating choline acetyltransferase and increasing norepinephrine contents in the brain regions [30]. Moreover, the administration of DJS results in an increase in plasma estrogen in the OVX rat model [31]. We supposed that memory primarily depends on hippocampal estrogen synthesis in an OVX model because it is difficult to synthesize estrogen in the ovaries. However, it is not yet clear whether DJS can synthesize estrogen in the hippocampus in an OVX model.

Thus, in this study, we explored whether the memory improvement effect of DJS is due to hippocampal estrogen synthesis and its related mechanisms focusing on synapse consolidation by assessing the expression of phosphorylated CREB (pCREB), SYN, and PSD-95 in an OVX model. We measured estrogen levels in primary hippocampal cells and the hippocampus of female mice under postmenopausal conditions after DJS treatment.

\section{Methods \\ Materials}

Neurobasal media (NM), B27, and penicillin-streptomycin $(\mathrm{P} / \mathrm{S})$ were purchased from Gibco Industries Inc. (Auckland, NZ). Rabbit monoclonal anti-pCREB and rabbit monoclonal anti-CREB were purchased from Santa Cruz Biotechnology, Inc. (Santa Cruz, CA, USA). Rabbit polyclonal anti-PSD-95 was purchased from Abcam (Cambridge, UK). Phosphate buffered saline (PBS), phosphatase inhibitor cocktail, 17 $\beta$ estradiol (EST), 2-methyl-2-butanol, 2,2,2-tribromoethanol and mouse monoclonal anti-SYN were purchased from Sigma-Aldrich (St. Louis, MO, USA). Anti-rabbit and mouse-horse radish peroxidase secondary antibodies were purchased from Assay Designs Inc. (Ann Arbor, MI, USA). The EST high sensitivity enzyme-linked immunosorbent assay (ELISA) kit was purchased from Enzo Life Sciences Inc. (Farmingdale, NY, USA). Other reagents for Western blotting were purchased from Bio-Rad Laboratories (Hercules, CA, USA).

\section{Preparation of DJS}

The water extract of DJS was the same as that used in our previous studies [32-34] in which it was standardized using paeoniflorin and albiflorin [32]. Briefly, we purchased individual dried herbs of DJS from Jung Do Herbal Drug Co. Ltd. (Seoul, Korea). Each herb was deposited at Department of Medicinal Herbology, College of Pharmacy, Kyung Hee University, Seoul, Korea. DJS consisted of $10 \mathrm{~g}$ Paeoniae Radix (Paeonia lactiflora Pallas, Paeoniaceae, number of voucher specimen: DBH14120301), 6 g Cnidii Rhizoma (Cnidium officinale Makino, Umbelliferae, number of voucher specimen: DBH16022422), $6 \mathrm{~g}$ Alismatis Rhizoma (Alisma orientale Juzepezuk, Alismataceae, number of voucher specimen: DBH16022411), $3 \mathrm{~g}$ Angelicae Gigantis Radix (Angelica gigas Nakai, Umbelliferae, number of voucher specimen: DBH16021001), $3 \mathrm{~g}$ Poria (Poria cocos Wolf, Polyporaceae, number of voucher specimen: DBH16022404), and 3 g Atractylodis Rhizoma Alba (Atractylodes macrocephala Koidzumi, Compositae, number of voucher specimen: DBH14111011). The mixed dried herbs were boiled with 10-fold distilled water for $2 \mathrm{~h}$ at $100{ }^{\circ} \mathrm{C}$ based on the way of decocting DJS in the clinical use. 
The suspension was filtered, lyophilized, yielding $20.16 \%$ of powder, and kept at $-20{ }^{\circ} \mathrm{C}$. This powder was dissolved in an appropriate vehicle before each experiment.

\section{Animals, surgery, and treatment}

Female ICR mice (age, 8 weeks; weight, 32-35 g) were purchased from Daehan Biolink (Eumseong, Korea) under specific pathogen free status. The animals were randomly divided and housed 10 mice per cage, had free access to water and food, and were maintained under constant temperature $\left(23 \pm 1{ }^{\circ} \mathrm{C}\right)$, humidity $(60 \pm 10 \%)$, and a $12 \mathrm{~h}$ light/dark cycle. The animals were treated and cared for in accordance with the Animal Care and Use Guidelines issued by Kyung Hee University, Korea. The experimental animal protocols were approved by the Institutional Animal Care and Use Committee of Kyung Hee University, Korea (KHUASP-15-010). After 1 week of acclimation, OVX was performed to remove the influence of ovary-synthesized estrogens under anesthetics using intraperitoneally injection of 2,2,2-tribromoethaol in 2-methyl-2-butanol. After OVX surgery, there were no adverse events in mice. Following a 2-week post-surgical recovery period, the mice were divided randomly into five groups $(n=10 /$ group for behavior tests to meet the statistical significance, total 50 mice): (1) the sham group (non-OVX and vehicle-treated), (2) the OVX group (OVX-lesioned and vehicle-treated group), (3) DJS $50 \mathrm{mg} / \mathrm{kg} /$ day group (OVX-lesioned and DJS $50 \mathrm{mg} / \mathrm{kg} /$ day; p.o.), (4) DJS $100 \mathrm{mg} / \mathrm{kg} /$ day group (OVX-lesioned and DIS $100 \mathrm{mg} / \mathrm{kg} /$ day; p.o.), and (5) EST group (OVX-lesioned and EST $200 \mu \mathrm{g} / \mathrm{kg} /$ day; i.p.). The DIS and EST groups were administrated once daily during 21 days and the sham and OVX groups were treated with the same volume of vehicle (Additional file 1).

\section{Novel object recognition test (NORT)}

The NORT was carried out in a black open field box $(45 \times 45 \times 50 \mathrm{~cm})$ as described previously [35]. Results are expressed as a percentage of novel object recognition time: Novel object recognition index $=[$ (time exploring novel object)/ (time exploring novel object + time exploring familiar object)] $\times 100$.

\section{Y-maze test}

The Y-maze test was carried out in a three-arm horizontal maze (each arm had a $120^{\circ}$ angle and was $40 \mathrm{~cm}$ long, $3 \mathrm{~cm}$ wide, and $12 \mathrm{~cm}$ high) as described previously [36]. The number of arm entries was recorded manually for each mouse over an 8 min period. Actual alternation was defined as entry into all three arms consecutively, such as $\mathrm{ABC}, \mathrm{CAB}$, or $\mathrm{BCA}$. Results are expressed as a percentage of spontaneous alternation using the following equation: $\%$ Spontaneous alternation $=[$ (number of alternations $) /$ (total arm entries -2$)] \times 100$.

\section{Brain tissue preparation}

The mice were decapitated after the behavioral tests, and their brains were collected rapidly and excised. Hippocampal tissues were stored at $-70{ }^{\circ} \mathrm{C}$ until the ELISA and western blot were performed.

\section{Cell culture}

Cell cultures were prepared from the hippocampi of 18day-old embryos from timed pregnant Sprague-Dawley rats (Daehan Biolink). The hippocampi were dissected, collected, dissociated, and plated in $60-\mathrm{mm}$ dishes at a density of $1 \times 10^{6}$ cells/dish. The cultures were maintained in a humidified incubator of $5 \% \mathrm{CO}_{2}$ at $37{ }^{\circ} \mathrm{C}$ in $\mathrm{NM}$ with $2 \mathrm{mM}$ glutamine, $2 \% \mathrm{~B} 27$, and $1 \% \mathrm{P} / \mathrm{S}$. After 3 days of incubation, the medium was replaced with a new medium. The cells were treated with DJS for $48 \mathrm{~h}$ on day 13 , and the supernatant was collected.

\section{Determination of EST using ELISA}

The concentration of EST was detected using the ELISA kit according to the manufacturer's instructions. Briefly, the supernatants ( $n=5$ /group) from homogenized hippocampi, hippocampal neurons, and standards were incubated on a pre-coated DxS immunoglobulin G immunoplate with EST conjugate and antibody overnight at $4{ }^{\circ} \mathrm{C}$. The plate was washed at least three times with buffer and incubated with para-nitrophenylphosphate substrate solution, which was catalyzed by alkaline phosphatase on the EST conjugate. After incubation for $1 \mathrm{~h}$, the stop solution was added to each well. The plate was read at $405 \mathrm{~nm}$ using a spectrophotometer (Versamax microplate reader; Molecular Devices, Sunnyvale, CA, USA) and concentrations of EST were determined in the sample solution using a EST standard calibration curve. Protein from the hippocampal samples was normalized using the Bio-Rad assay for total protein determination.

\section{Western blotting}

Homogenized hippocampal lysates ( $n=5 /$ group) were separated on $12 \%$ sodium dodecyl sulfatepolyacrylamide gel electrophoresis and transferred to a polyvinylidene fluoride membrane. The membranes were incubated with $5 \%$ skim milk in Tween-20 for $1 \mathrm{~h}$ and then with primary antibodies (1:5000 of pCREB, and CREB and 1:1000 of SYN, PSD-95, and $\beta$-actin) overnight at $4{ }^{\circ} \mathrm{C}$, followed by incubation with horseradish peroxidase-conjugated secondary antibodies for $1 \mathrm{~h}$. Immunoreactive-bands were detected using an enhanced chemiluminescence detection kit, and the LAS-4000 mini system (Fujifilm Corp., Tokyo, Japan) was used for visualization. Bands intensity was normalized to the non-phospho form band or the $\beta$-actin band using Multi Gauge software (Fujifilm Corp.). 


\section{Statistical analysis}

All statistical parameters were calculated using GraphPad Prism software (ver. 5.0; GraphPad Software Inc., San Diego, CA, USA). Values are expressed as means \pm standard error of the mean. Results were analyzed by one-way analysis of variance (ANOVA) followed by Tukey's post-hoc test. A p-value $<0.05$ was considered significant.

\section{Results}

DJS stimulates hippocampal estrogen synthesis both in the primary cells and in the mouse hippocampus

Increasing evidence indicates that hippocampus-derived estrogen contributes to promoting synaptic plasticity and neuroprotective actions rather than estrogen synthesized from gonads [37]. We investigated whether DJS affects hippocampal estrogen synthesis in vitro and in vivo. The treatment of primary hippocampal cells with $10 \mu \mathrm{g} / \mathrm{mL}$ DJS for $48 \mathrm{~h}$ increased the levels of EST compared to the untreated group (Fig. 1A). Furthermore, to confirm the effects of DJS in vivo, we administered DJS at 50 and $100 \mathrm{mg} / \mathrm{kg} /$ day orally to mice for 21 days starting from 2 weeks after OVX. The levels of hippocampal EST in the OVX group were significantly lower than those in the sham group but were significantly increased by DJS treatment at $100 \mathrm{mg} / \mathrm{kg} /$ day (Fig. 1B).

\section{DJS ameliorates OVX-induced memory impairments in mice}

To examine the effects of DJS-stimulated hippocampal estrogen on memory function, we performed NORT and Y-maze test, which measured cognitive function and spatial working memory of rodents [38]. In NORT, the time spent exploring a novel object in the OVX group was shorter than that of the sham group (Fig. 2A). Both the DJS treatments at 50 and $100 \mathrm{mg} / \mathrm{kg} /$ day led to increased time of exploring the novel object during the test session compared to that of the OVX group. In the Y-maze test, the percentage of spontaneous alternation in the OVX group was significantly lower than that of the sham group (Fig. 2B). The DJS-treated group with $100 \mathrm{mg} / \mathrm{kg} /$ day significantly showed a higher percentage of alternation than that of the OVX group. Together, we show that the DJS attenuated memory impairment induced by estrogen deprivation in mice.

\section{DJS induces CREB phosphorylation in OVX mice}

Hippocampal estrogen facilitates phosphorylation of CREB, which is a nuclear protein that is required for memory formation and consolidation [39, 40]. We investigated whether DJS activates CREB expression under estrogen deprivation status. The ratio of pCREB/CREB in the OVX group was significantly reduced compared with those in the sham group; however, the DJS at $100 \mathrm{mg} / \mathrm{kg} /$ day significantly restored this ratio with similar to EST-treated group (Fig. 3).

\section{DJS restores OVX-induced synaptic loss in the mouse hippocampus}

It has been reported that estrogen modulates spine synapse formation in the hippocampal pyramidal neurons, which are regions for a key role in learning and memory [41]. To investigate the ameliorating effects of DJS on synaptic damage induced by OVX, we measured the expression levels of presynaptic (SYN) and postsynaptic (PSD-95) in the mouse hippocampus. We found that treatment of DJS at $100 \mathrm{mg} / \mathrm{kg}$ also significantly elevated the expression levels of SYN and PSD-95 compared to those in the OVX group (Fig. 4).

\section{Discussion}

In the present study, we showed that DJS attenuated memory deficits and facilitated CREB and synaptic
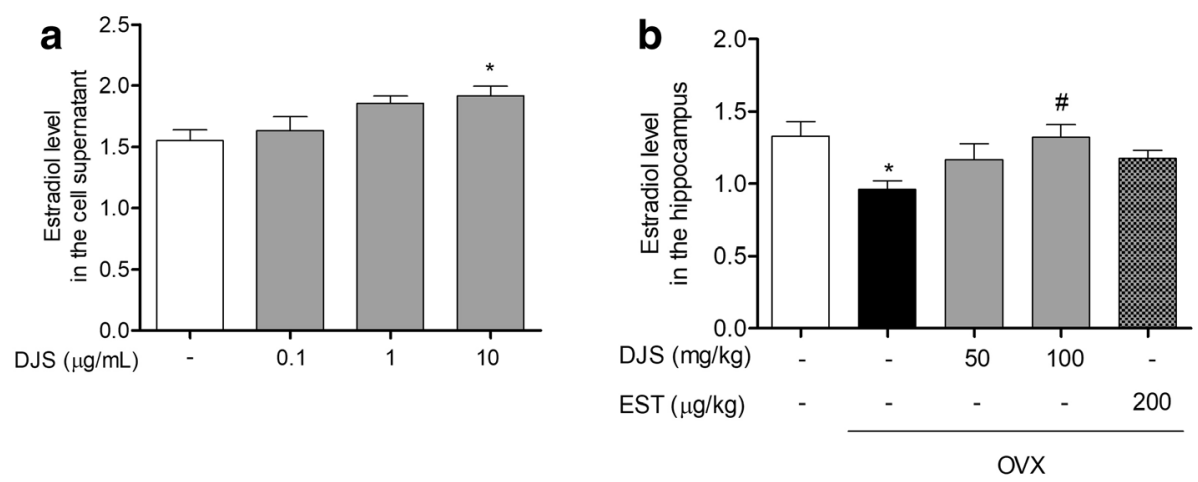

Fig. 1 Effects of DJS on hippocampal estrogen synthesis in primary hippocampal cells and hippocampus of OVX mice. Rat primary hippocampal cells were treated with the DJS for $48 \mathrm{~h}$. Female mice were administrated with DJS at the concentration of 50 and $100 \mathrm{mg} / \mathrm{kg} / \mathrm{day}$ and EST at $200 \mu \mathrm{g} / \mathrm{kg} /$ day for 21 days after OVX surgery. Then, EST levels in the supernatant of cell culture (a) and hippocampus (b) were measured by ELISA. Values are means \pm standard error. ${ }^{*} p<0.05$ compared with the sham group, \#p $<0.05$ compared with the OVX group 

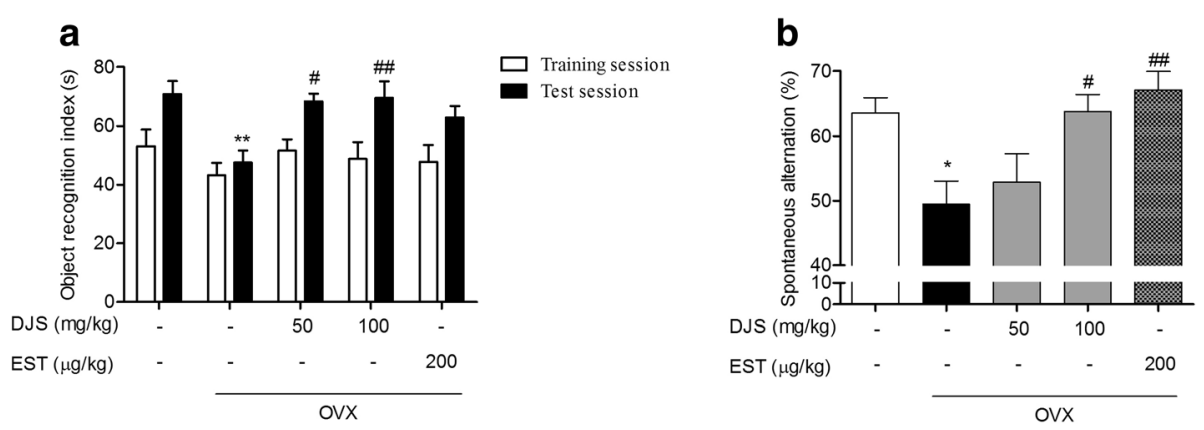

Fig. 2 Effects of DJS on OVX-induced cognitive decline. OVX-induced cognitive decline was determined by novel object recognition test and Y-maze test. Times spent on novel and familiar objects were measured on the novel object recognition test (a). Spontaneous alternation behavior and the number of arm entries wre measured on the $Y$-maze test (b). ${ }^{*} p<0.05$ and ${ }^{* *} p<0.01$ compared with the sham group, \#p $<0.05$ and \#\#p $<0.01$ compared with the OVX group

activation via stimulation of hippocampal estrogen synthesis in OVX mice. Growing evidence indicates that estrogen plays a key role in memory function by enhancing the expression of synaptic proteins and LTPmediated synaptic plasticity in the hippocampus $[14,23$, 42]. It was first demonstrated that estrogen can be synthesized de novo in the hippocampus by Fester et al. and hippocampus-derived estrogen is more closely linked to synaptic plasticity than that derived from the gonads $[37,43]$. It has been reported that DJS increases estrogen levels in the blood of senescence accelerated mouseprone 8 mice and OVX rats $[31,44]$. The dose and

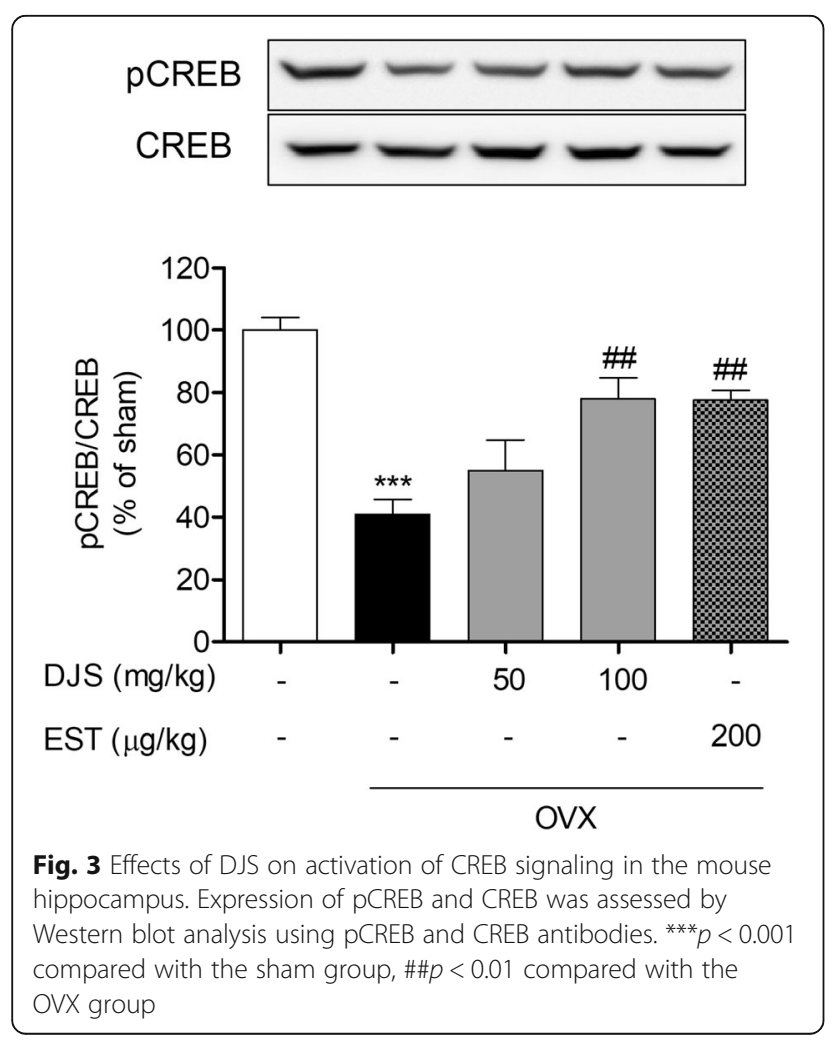

duration of DJS administration in these studies were much higher and longer than in our study [31, 44]. Actually, the treatment of DJS in our study showed no change in serum estrogen levels (Additional file 2). Thus, we assume that the estrogen levels in the blood may depend on the dose and duration of DJS treatment.

To investigate the mechanism underlying DJS amelioration of OVX-induced memory deficits in mice, we analyzed the expression levels of PCREB, SYN, and PSD-95 in the hippocampus. The phosphorylation of CREB stimulates the synthesis of proteins such as SYN and PSD-95, which are related to synapse formation, resulting in memory enhancement [45-47]. SYN is an essential membrane glycoprotein that exists in presynaptic vesicles of neurons [48]. SYN as presynaptic marker is closely connected with cognitive function, and altering of this marker is important for synaptic connectivity and plasticity [49]. It has been reported that change of estrogen levels can affect SYN expression [41]. We confirmed that DJS could induce SYN expression via the increase of hippocampal estrogen levels, resulting amelioration of memory impairment in OVX mice. These results suggest that SYN works as a mediator of ameliorating memory deficits after DJS treatment in OVX mice.

Astrocytes, which are the most abundant glial cells in the mammalian brain, involve in the metabolic control of estrogen and has the potential to mediate neuroprotective actions of estrogen [50-52]. Thus, astrocyte activation is closely associated with estrogen signaling in brain. We found that the number of glial fibrillary acidic protein (GFAP) as an astrocyte marker-positive cells in the OVX group was significantly reduced compared with those in the sham group, however, the DJS treatment significantly restored the number with similar to ESTtreated group (Additional file 3). These results suggest that DJS treatment has the possibility that induce estrogen metabolism in astrocytes as well as in neurons. 

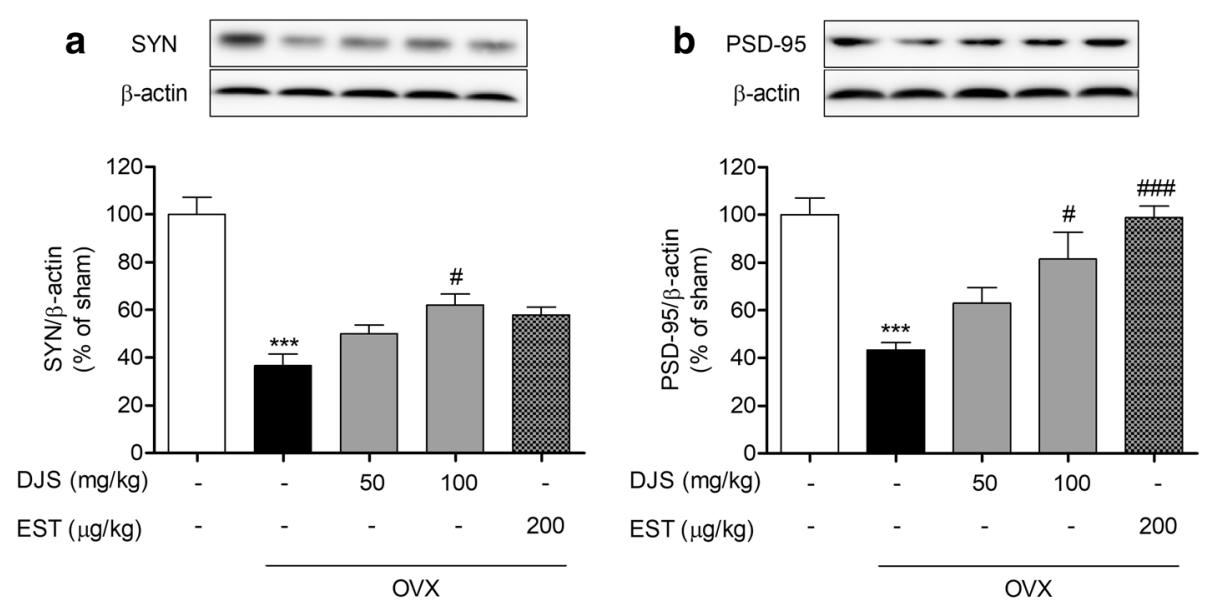

Fig. 4 Effects of DJS on OVX-induced synaptic loss in the mouse hippocampus. Expression of synaptophysin (SYN) and PSD-95 was assessed by Western blot analysis using SYN (a) and PSD-95 (b) antibodies. ${ }^{* *} \mathrm{p}<0.001$ compared with the sham group, \#p $<0.05$ and \#\#\# $<0.001$ compared with the OVX group

In this study, we demonstrated that DJS significantly ameliorated the memory deficits in OVX mice, by increasing the memory-related factors such as CREB and synaptic proteins via inducing the hippocampal estrogen synthesis. Previous studies have shown that hippocampal estrogen increases memory function via regulation of presynaptic and postsynaptic protein expression [23, 53]. Kato et al. reported that all sex steroids including estrogen are much higher in the hippocampus than in the blood [54]. Several studies have also shown that hippocampus-derived estrogen has a key role in synaptic plasticity, increasing spine synapse and expression of synaptic proteins, thereby improving memory function $[14,18,55]$. Given the evidence on the role of hippocampal estrogen, it is important that hippocampal estrogen shows the higher contribution to memory function than peripheral estrogen, and DJS can upregulate hippocampal estrogen.

\section{Conclusion}

In conclusion, our results demonstrated that DJS ameliorated OVX-induced memory dysfunction via inducing hippocampal estrogen synthesis, elevating the expression of CREB and synaptic proteins. We also suggest that DJS could be a potential candidate for preventing and treating memory decline in postmenopausal women.

\section{Additional files}

Additional file 1: Experimental design for surgery, drug administration and behavioral test (PDF $43 \mathrm{~kb}$ )

Additional file 2: DJS did not induce serum estrogen synthesis in OVX mice (PDF $132 \mathrm{~kb}$ )
Additional file 3: Effects of DJS on OVX-induced decrease of astrocyte activation in the mouse hippocampus (PDF $90 \mathrm{~kb}$ )

Additional file 4: Additional information (PDF $32 \mathrm{~kb}$ )

\section{Abbreviations \\ CREB: CAMP response element-binding protein; DJS: Dangguijakyak-san; ELISA: Enzyme-linked immunosorbent assay; ERK: Extracellular-signal- regulated kinase; EST: 17ß-estradiol; LTP: Long-term potentiation; NM: Neurobasal media; NORT: Novel object recognition test; OVX: Ovariectomized; P/S: Penicillin-streptomycin; pCREB: Phosphorylated CREB; PSD-95: Postsynaptic density protein-95; SYN: Synaptophysin}

\section{Acknowledgements}

Not applicable.

\section{Funding}

This study was supported by Bio-Synergy Research Project (National Research Foundation [NRF]-2012M3A9C4048795) of the Ministry of Science, ICT, and Future Planning (MSIP) through the NRF.

Availability of data and materials

All data generated or analyzed during this study are included in this published article and its Additional file 1-4 and Additional information.

Authors' contributions

DSH, HCK, and MSO designed and coordinated the study. HGK and NKK performed the experiment and acquired the data. JGC, NKK, and MSO wrote the paper. All the authors participated in discussion of the results and reviewed the final draft. All authors read and approved the final manuscript.

Ethics approval and consent to participate

All procedures involving animals were conducted in accordance with the Animal Care and Use Guidelines issued by Kyung Hee University, Korea. The experimental animal protocols were approved by the Institutional Animal Care and Use Committee of Kyung Hee University, Korea (KHUASP-15-010).

Consent for publication

Not applicable.

Competing interests

The authors declare that they have no competing interests. 


\section{Publisher's Note}

Springer Nature remains neutral with regard to jurisdictional claims in published maps and institutional affiliations.

\section{Author details}

'Department of Oriental Gynecology, College of Oriental Medicine, Kyung Hee University, 26, Kyungheedae-ro, Dongdaemun-gu, Seoul 02447, Republic of Korea. ${ }^{2}$ Department of Life and Nanopharmaceutical Sciences, Graduate School, Kyung Hee University, 26, Kyungheedae-ro, Dongdaemun-gu, Seoul 02447, Republic of Korea. ${ }^{3}$ Department of Oriental Pharmaceutical Science, College of Pharmacy and Kyung Hee East-West Pharmaceutical Research Institute, Kyung Hee University, 26, Kyungheedae-ro, Dongdaemun-gu, Seoul 02447, Republic of Korea. ${ }^{4}$ Department of Herbal Pharmacology, College of Korean Medicine, Kyung Hee University, 26, Kyungheedae-ro,

Dongdaemun-gu, Seoul 02447, Republic of Korea.

Received: 13 April 2017 Accepted: 17 November 2017

Published online: 25 November 2017

\section{References}

1. Luine VN, Richards ST, Wu W, Beck KD. Estradiol enhances learning and memory in a spatial memory task and effects levels of monoaminergic neurotransmitters. Horm Behav. 1998;34(2):149-62.

2. Smith CC, McMahon LL. Estrogen-induced increase in the magnitude of long-term potentiation occurs only when the ratio of NMDA transmission to AMPA transmission is increased. J Neurosci. 2005:25(34):7780-91.

3. Kramar EA, Chen LY, Brandon NJ, Rex CS, Liu F, Gall CM, Lynch G. Cytoskeletal changes underlie estrogen's acute effects on synaptic transmission and plasticity. J Neurosci. 2009;29(41):12982-93.

4. Vedder LC, Smith CC, Flannigan AE, McMahon LL. Estradiol-induced increase in novel object recognition requires hippocampal NR2B-containing NMDA receptors. Hippocampus. 2013;23(1):108-15.

5. Frick KM. Molecular mechanisms underlying the memory-enhancing effects of estradiol. Horm Behav. 2015;74:4-18.

6. McEwen BS, Akama KT, Spencer-Segal JL, Milner TA, Waters EM. Estrogen effects on the brain: actions beyond the hypothalamus via novel mechanisms. Behav Neurosci. 2012:126(1):4-16.

7. McEwen B, Akama K, Alves S, Brake WG, Bulloch K, Lee S, Li C, Yuen G, Milner TA. Tracking the estrogen receptor in neurons: implications for estrogen-induced synapse formation. Proc Natl Acad Sci U S A. 2001;98(13): 7093-100.

8. Kelly A, Laroche S, Davis S. Activation of mitogen-activated protein kinase/ extracellular signal-regulated kinase in hippocampal circuitry is required for consolidation and reconsolidation of recognition memory. J Neurosci. 2003; 23(12):5354-60.

9. Wade CB, Dorsa DM. Estrogen activation of cyclic adenosine 5'monophosphate response element-mediated transcription requires the extracellularly regulated kinase/mitogen-activated protein kinase pathway. Endocrinology. 2003;144(3):832-8.

10. Yokomaku D, Numakawa T, Numakawa Y, Suzuki S, Matsumoto T, Adachi N, Nishio C, Taguchi T, Hatanaka H. Estrogen enhances depolarization-induced glutamate release through activation of phosphatidylinositol 3-kinase and mitogen-activated protein kinase in cultured hippocampal neurons. Mol Endocrinol. 2003;17(5):831-44.

11. Szego EM, Barabas K, Balog J, Szilagyi N, Korach KS, Juhasz G, Abraham IM. Estrogen induces estrogen receptor alpha-dependent CAMP response element-binding protein phosphorylation via mitogen activated protein kinase pathway in basal forebrain cholinergic neurons in vivo. J Neurosci. 2006;26(15):4104-10

12. Akama KT, McEwen BS. Estrogen stimulates postsynaptic density-95 rapid protein synthesis via the Akt/protein kinase B pathway. J Neurosci. 2003; 23(6):2333-9.

13. Frick KM, Fernandez SM, Bulinski SC. Estrogen replacement improves spatial reference memory and increases hippocampal synaptophysin in aged female mice. Neuroscience. 2002;115(2):547-58.

14. Kretz O, Fester L, Wehrenberg U, Zhou L, Brauckmann S, Zhao S, Prange-Kiel J, Naumann T, Jarry H, Frotscher M, et al. Hippocampal synapses depend on hippocampal estrogen synthesis. J Neurosci. 2004;24(26):5913-21.

15. Woolley CS, McEwen BS. Roles of estradiol and progesterone in regulation of hippocampal dendritic spine density during the estrous cycle in the rat. J Comp Neurol. 1993;336(2):293-306.
16. MacLusky NJ, Luine VN, Hajszan T, Leranth C. The 17alpha and 17beta isomers of estradiol both induce rapid spine synapse formation in the CA1 hippocampal subfield of ovariectomized female rats. Endocrinology. 2005;146(1):287-93.

17. Chen JR, Yan YT, Wang TJ, Chen LJ, Wang YJ, Tseng GF. Gonadal hormones modulate the dendritic spine densities of primary cortical pyramidal neurons in adult female rat. Cereb Cortex. 2009;19(11):2719-27.

18. Tuscher JJ, Szinte JS, Starrett JR, Krentzel AA, Fortress AM, Remage-Healey L, Frick KM. Inhibition of local estrogen synthesis in the hippocampus impairs hippocampal memory consolidation in ovariectomized female mice. Horm Behav. 2016;83:60-7.

19. Do Rego JL, Seong JY, Burel D, Leprince J, Luu-The V, Tsutsui K, Tonon MC, Pelletier G, Vaudry H. Neurosteroid biosynthesis: enzymatic pathways and neuroendocrine regulation by neurotransmitters and neuropeptides. Front Neuroendocrinol. 2009;30(3):259-301.

20. Cui J, Shen Y, Li R. Estrogen synthesis and signaling pathways during aging: from periphery to brain. Trends Mol Med. 2013;19(3):197-209.

21. Yague JG, Azcoitia I, DeFelipe J, Garcia-Segura LM, Munoz A. Aromatase expression in the normal and epileptic human hippocampus. Brain Res. 2010;1315:41-52.

22. Gottfried-Blackmore A, Sierra A, Jellinck PH, McEwen BS, Bulloch K. Brain microglia express steroid-converting enzymes in the mouse. J Steroid Biochem Mol Biol. 2008;109(1-2):96-107.

23. Prange-Kiel J, Fester L, Zhou L, Lauke H, Carretero J, Rune GM. Inhibition of hippocampal estrogen synthesis causes region-specific downregulation of synaptic protein expression in hippocampal neurons. Hippocampus. 2006; 16(5):464-71.

24. Yun SH, Park KA, Kwon S, Woolley CS, Sullivan PM, Pasternak JF, Trommer BL. Estradiol enhances long term potentiation in hippocampal slices from aged apoE4-TR mice. Hippocampus. 2007;17(12):1153-7.

25. Zhou L, Fester L, Haghshenas S, de Vrese X, von Hacht R, Gloger S, Brandt $\mathrm{N}$, Bader M, Vollmer G, Rune GM. Oestradiol-induced synapse formation in the female hippocampus: roles of oestrogen receptor subtypes. J Neuroendocrinol. 2014;26(7):439-47.

26. Hagino N. An overview of Kampo medicine: Toki-Shakuyaku-san (TJ-23). Phytother Res. 1993;7(6):391-4.

27. Yi JH, Hye Jin P, Beak SJ, Lee S, Jung JW, Kim BC, Ryu JH, Kim DH. DangguiJakyak-san enhances hippocampal long-term potentiation through the ERK CREB/BDNF cascade. J Ethnopharmacol. 2015:175:481-9.

28. Song MD, Kim DH, Kim JM, Lee HE, Park SJ, Ryu JH, Lew JH. DangguiJakyak-san ameliorates memory impairment and increase neurogenesis induced by transient forebrain ischemia in mice. BMC Complement Altern Med. 2013;13:324

29. Hu ZY, Liu G, Yuan H, Yang S, Zhou WX, Zhang YX, Qiao SY. DangguiShaoyao-san and its active fraction JD-30 improve Abeta-induced spatial recognition deficits in mice. J Ethnopharmacol. 2010;128(2):365-72.

30. Toriizuka K, Hou P, Yabe T, lijima K, Hanawa T, Cyong JC. Effects of Kampo medicine, Toki-shakuyaku-san (tang-Kuei-Shao-Yao-san), on choline acetyltransferase activity and norepinephrine contents in brain regions, and mitogenic activity of splenic lymphocytes in ovariectomized mice. J Ethnopharmacol. 2000;71(1-2):133-43.

31. Chung MH, Suzuki S, Nishihara T, Hattori M. Estrogenic effects of a Kampo formula, Tokishakuyakusan, in parous ovariectomized rats. Biol Pharm Bull. 2008;(6):31, 1145-1149.

32. Hwang DS, Kim HG, Kwon HJ, Cho JH, Lee CH, Lee JM, Jang JB, Kim YS, Lee KS, Oh MS. Dangguijakyak-san, a medicinal herbal formula, protects dopaminergic neurons from 6-hydroxydopamine-induced neurotoxicity. J Ethnopharmacol. 2011;133(2):934-9.

33. Hwang DS, Kim HG, Jang JB, Oh MS. Dangguijakyak-san protects against 1Methyl-4-phenyl-1,2,3,6,-tetrahydropyridine-induced neuronal damage via anti-inflammatory action. Evid Based Complement Alternat Med. 2013; 2013:976270.

34. Lee JM, Hwang DS, Kim HG, Lee CH, Oh MS. Dangguijakyak-san protects dopamine neurons against 1-methyl-4-phenyl-1,2,3,6-tetrahydropyridineinduced neurotoxicity under postmenopausal conditions. J Ethnopharmacol. 2012;139(3):883-8

35. Huh E, Kim HG, Park H, Kang MS, Lee B, Oh MS. Houttuynia Cordata improves cognitive deficits in cholinergic dysfunction Alzheimer's diseaselike models. Biomol Ther (Seoul). 2014;22(3):176-83.

36. Lee $Y$, Kim J, Jang S, Oh S. Administration of Phytoceramide Enhances Memory and Upregulates the expression of pCREB and BDNF in hippocampus of mice. Biomol Ther (Seoul). 2013;21(3):229-33. 
37. Fester L, Prange-Kiel J, Jarry H, Rune GM. Estrogen synthesis in the hippocampus. Cell Tissue Res. 2011;345(3):285-94.

38. Wolf A, Bauer B, Abner EL, Ashkenazy-Frolinger T, Hartz AM. A comprehensive behavioral test battery to assess learning and memory in 12956/Tg2576 mice. PLoS One. 2016;11(1):e0147733.

39. Silva AJ, Kogan JH, Frankland PW, Kida S. CREB and memory. Annu Rev Neurosci. 1998;21:127-48.

40. Lee SJ, Campomanes CR, Sikat PT, Greenfield AT, Allen PB, McEwen BS. Estrogen induces phosphorylation of cyclic AMP response element binding ( $p C R E B)$ in primary hippocampal cells in a time-dependent manner. Neuroscience. 2004;124(3):549-60.

41. Hara Y, Waters EM, McEwen BS, Morrison JH. Estrogen effects on cognitive and synaptic health over the Lifecourse. Physiol Rev. 2015;95(3):785-807.

42. Stelly CE, Cronin J, Daniel JM, Schrader LA. Long-term oestradiol treatment enhances hippocampal synaptic plasticity that is dependent on muscarinic acetylcholine receptors in ovariectomised female rats. J Neuroendocrinol. 2012;24(6):887-96.

43. Prange-Kiel J, Wehrenberg U, Jarry H, Rune GM. Para/autocrine regulation of estrogen receptors in hippocampal neurons. Hippocampus. 2003;13(2):22634.

44. Huang Y, Hu ZY, Yuan H, Shu L, Liu G, Qiao SY, Sun L, Zhou WX, Zhang YX. Danggui-Shaoyao-san improves learning and memory in female SAMP8 via modulation of Estradiol. Evid Based Complement Alternat Med. 2014;2014: 327294.

45. Bernabeu R, Bevilaqua L, Ardenghi P, Bromberg E, Schmitz P, Bianchin M, Izquierdo I, Medina JH. Involvement of hippocampal CAMP/CAMPdependent protein kinase signaling pathways in a late memory consolidation phase of aversively motivated learning in rats. Proc Natl Acad Sci U S A. 1997;94(13):7041-6.

46. Bourtchuladze R, Frenguelli B, Blendy J, Cioffi D, Schutz G, Silva AJ. Deficient long-term memory in mice with a targeted mutation of the CAMPresponsive element-binding protein. Cell. 1994;79(1):59-68.

47. Mizuno M, Yamada K, Maekawa N, Saito K, Seishima M, Nabeshima T. CREB phosphorylation as a molecular marker of memory processing in the hippocampus for spatial learning. Behav Brain Res. 2002;133(2):135-41.

48. Wiedenmann B, Franke WW, Kuhn C, Moll R, Gould VE. Synaptophysin: a marker protein for neuroendocrine cells and neoplasms. Proc Natl Acad Sci U S A. 1986;83(10):3500-4.

49. Stockert JC, Blazquez-Castro A, Canete M, Horobin RW, Villanueva A. MTT assay for cell viability: intracellular localization of the formazan product is in lipid droplets. Acta Histochem. 2012;114(8):785-96.

50. Garcia-Ovejero D, Azcoitia I, Doncarlos LL, Melcangi RC, Garcia-Segura LM. Glia-neuron crosstalk in the neuroprotective mechanisms of sex steroid hormones. Brain Res Brain Res Rev. 2005:48(2):273-86.

51. Pawlak J, Karolczak M, Krust A, Chambon P, Beyer C. Estrogen receptoralpha is associated with the plasma membrane of astrocytes and coupled to the MAP/Src-kinase pathway. Glia. 2005;50(3):270-5.

52. Struble RG, Nathan BP, Cady C, Cheng X, McAsey M. Estradiol regulation of astroglia and apolipoprotein $\mathrm{E}$ : an important role in neuronal regeneration. Exp Gerontol. 2007:42(1-2):54-63.

53. Smejkalova T, Woolley CS. Estradiol acutely potentiates hippocampal excitatory synaptic transmission through a presynaptic mechanism. J Neurosci. 2010;30(48):16137-48.

54. Kato A, Hojo Y, Higo S, Komatsuzaki Y, Murakami G, Yoshino H, Uebayashi M, Kawato $S$. Female hippocampal estrogens have a significant correlation with cyclic fluctuation of hippocampal spines. Front Neural Circuits. 2013;7:149.

55. Hojo Y, Murakami G, Mukai H, Higo S, Hatanaka Y, Ogiue--keda M, Ishii H, Kimoto T, Kawato $S$. Estrogen synthesis in the brain-role in synaptic plasticity and memory. Mol Cell Endocrinol. 2008;290(1-2):31-43.

\section{Submit your next manuscript to BioMed Central and we will help you at every step:}

- We accept pre-submission inquiries

- Our selector tool helps you to find the most relevant journal

- We provide round the clock customer support

- Convenient online submission

- Thorough peer review

- Inclusion in PubMed and all major indexing services

- Maximum visibility for your research

Submit your manuscript at www.biomedcentral.com/submit
Biomed Central 